\title{
Energy Detection Using NI USRP 2920
}

\author{
Sowmiya $\mathbf{M}^{1}$, Sangeetha $\mathbf{M}^{2}$ \\ ${ }^{1}$ Assistant Professor, Department of ECE, PSG Institute of Technology and Applied Research, Coimbatore, India \\ ${ }^{2}$ Assistant Professor, Department of EEE, Dr. Mahalingam College of Engineering and Technology, Pollachi, India
}

\begin{abstract}
The idea of this paper is to detect the presence of primary users to avoid interference with secondary users in cognitive radio networks. The performance of Cognitive Radio (CR) systems with Energy Detection with modulation is analysed and CR is implemented using Universal Software Radio Peripheral National Instruments 2920. It is the widely used Software Defined Radio kit for learning digital wireless communication system.
\end{abstract}

Keywords: Cognitive Radio, Spectrum sensing, Energy Detection, USRP, LabVIEW

\section{Introduction}

Cognitive radio (CR) is a form of wireless communication in which a transceiver can intelligently detect the communication channels in use and which are allocated to secondary users [3]. The term Cognitive Radio is also promoted by Mitola. The available radio-frequency (RF) spectrum is utilized while minimizing interference to other users. CR is a technology involving software defined radio (SDR) as applied to spread spectrum communications. As defined by FCC, Cognitive radio is a radio or system that senses its operational electromagnetic environment and can dynamically and autonomously adjust its radio operating parameters to modify system operation, such as maximize throughput, mitigate interference, facilitate interoperability and access secondary markets [4].

Cognitive radio has the ability of a transceiver to determine the geographic location, identify and authorize its user, sense neighbouring wireless devices in operation and adjust output power and modulation characteristics. In cognitive radio, two types of users are considered primary users and secondary users. The primary users can be defined as the licensed users who are having high priority over a specific part of the spectrum. The secondary users are unlicensed users and are given low priority over spectrum usage. The secondary users have to exploit the spectrum such that they do not cause interference to primary users. Therefore secondary users need to have cognitive radio capabilities. It helps to check whether the spectrum is occupied by primary users or not. Two primary objectives are highly reliable communication whenever and wherever needed and efficient utilization of the radio spectrum [3].

A basic cognitive cycle comprises of following three basic tasks:

- Spectrum Sensing

- Spectrum Analysis

- Spectrum Decision Making

Cognitive radio has been used in various fields such as Disaster management, Public safety, and Emergency network. A public safety worker is increasingly equipped with mobile and smart devices are wirelessly connected to improve their efficiency, visibility, and have ability to communicate with central command, co-workers and other agencies. Radio frequencies allocated for Public Safety [2] use have become highly congested in many, especially urban areas. Cognitive radio is identified as one of emerging technologies to increase efficiency and effectiveness of spectrum usage. There are many test beds available for building cognitive radio such as USRP, WARP [12], [13] etc.

In this paper, the QPSK modulated signal is received and energy is analysed to detect the presence of primary users using USRP NI 2920.

Section 2 gives the overview of the spectrum sensing techniques. Section 3 describes the USRP NI 2920. Section 4 gives the result and its analysis and conclusion is given in Section 5 .

\section{Spectrum Sensing}

One main challenge to the Cognitive radio is spectrum sensing. Spectrum sensing is the task of obtaining awareness about the spectrum usage and existence of primary users in a geographical area [10]. Generally, spectrum sensing is considered as measuring the spectral content or radio frequency energy over the spectrum. Spectrum sensing helps to find spectrum hole, allowing unlicensed user to use vacant licensed band.

Consider two parameters $\mathrm{H}_{0}$ and $\mathrm{H}_{1}$.

$\mathrm{H}_{0}$ : Primary user is absent

$\mathrm{H}_{1}$ : Primary user is present

\subsection{Performance Metrics}

The detection algorithm performance is evaluated with two probabilities.

- Probability of Detection $\left(P_{D}\right)$

- Probability of False Alarm $\left(P_{F}\right)$.

(a) Probability of Detection $\left(\mathbf{P}_{D}\right)$

$\mathrm{P}_{\mathrm{D}}$ is the probability that the receiver detects the signal when it is truly present in the specified spectrum. A large detection probability is desired. Its expression is given by

$$
P_{D}=P_{r}\left(Y>\lambda_{t h} \mid H 1\right)
$$




\section{International Journal of Science and Research (IJSR) \\ ISSN (Online): 2319-7064}

Index Copernicus Value (2013): 6.14 | Impact Factor (2015): 6.391

where $\lambda_{t h}$ is the threshold chosen as a balance between $P_{D}$ and $P_{F}$.

(b) Probability of False Alarm $\left(\mathbf{P}_{F}\right)$

$\mathrm{P}_{\mathrm{F}}$ is the probability that the system incorrectly detects the signal when it is actually not present. $P_{F}$ should be kept as small as possible in order to prevent underutilization of transmission opportunities. It can be expressed as

$$
\mathrm{P}_{\mathrm{F}}=\mathrm{P}_{\mathrm{r}}\left(\mathrm{Y}>\lambda_{\mathrm{th}} / \mathrm{H}_{0}\right)
$$

\section{(c) Probability of Miss Detection $\left(\mathbf{P}_{M}\right)$}

$\mathrm{P}_{\mathrm{M}}$ is the probability that the receiver does not detect the signal when it is actually present in the actual spectrum. Its expression is given by

$$
P_{M}=1-P_{D}
$$

\section{(d) Receiver Operating Characteristic (ROC)}

ROC is a graph of Probability of False alarm versus Probability of Detection. It explores the relationship between sensitivity $\left(P_{D}\right)$ and specificity $\left(P_{F}\right)$ for various threshold values helping to choose an optimal threshold.

\section{(e) Complementary ROC}

Complementary ROC is the graph between Probability of False alarm versus Probability of Miss Detection.

Three methods of spectrum sensing are Transmitter Detection, Receiver Detection, and Interference Temperature Management [5].Transmitter detection is important as it is widely used and there are many types of transmitter detection methods such as Energy detection, Cyclostationary based detection and Matched filter detection [7]. Table 1 shows the comparison of spectrum sensing techniques present in transmitter detection interms of complexity, accuracy, sensing time.

Table 1: Comparison of Spectrum Sensing Techniques

\begin{tabular}{|l|c|c|c|}
\hline & Energy Detection & Cyclostationary & Matched Filtering \\
\hline Complexity & Low & High & High \\
\hline Sensing Time & High & High & Less \\
\hline Accuracy & Low & Medium & High \\
\hline
\end{tabular}

In this paper, Energy detection is considered as it is advantageous over other methods because of its simplicity in implementation.

\subsection{Energy Detection}

Energy detection is the most common way of spectrum sensing due to the lower computation required and no need of any knowledge about the possible signal. The detection could be done in time or frequency domain. Time domain implementation consists of averaging the square of the signal as shown in fig.1. Frequency domain requires an FFT whose size $\mathrm{N}$ improves frequency resolution helping narrow band signal detection and later averaging over the observation time as shown in fig 2. Frequency domain gives a second degree of freedom to improve the algorithm [10]. Finally in both cases, the result is compared with the threshold.

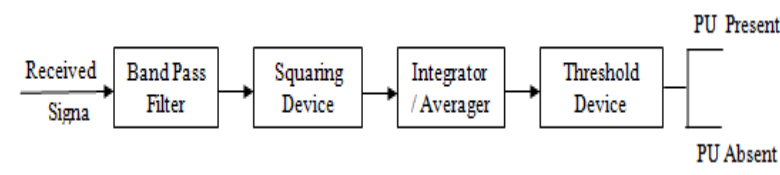

Figure 1: Time domain Energy Detector

The band pass filter selects the center frequency ' $\mathrm{fs}$ ' and band width of interest ' $W$ '. The Squaring Device which follows the filter measures the received energy. Then, the integrator determines the observation interval, ' $T$ '. The output of the integrator is compared with the threshold $\lambda_{\text {th }}$ which decides the presence or absence of the primary user.

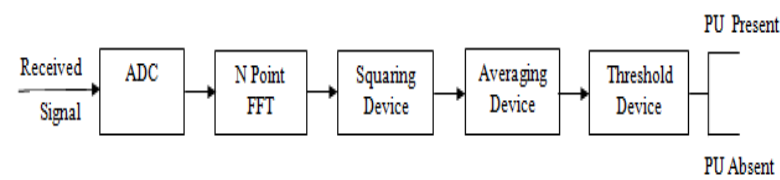

Figure 2: Frequency domain Energy Detector

In Energy detection method, the presence of primary user is detected by comparing the output of the energy detector $(\mathrm{M})$ with a threshold ( $\lambda$ th) which depends on the noise [10].

In discrete domain, the received signal can be represented as

$$
\mathrm{x}(\mathrm{n})=\left\{\begin{array}{l}
\mathrm{w}(\mathrm{n}) \mathrm{H}_{0} \\
\mathrm{~h} * \mathrm{~s}(\mathrm{n})+\mathrm{w}(\mathrm{n}) \mathrm{H}_{1}
\end{array}\right.
$$

where $\mathrm{x}(\mathrm{n})$ is the received signal, $\mathrm{w}(\mathrm{n})$ is the AWGN, $\mathrm{h}$ is the amplitude gain of the channel, and $s(n)$ is the transmitted signal. When there is no transmission by the primary user, the value of $\mathrm{s}(\mathrm{n})=0 . \mathrm{H}_{1}$ and $\mathrm{H}_{0}$ are the two hypothesis formulated for the existence or non-existence of primary transmitter respectively. The Decision Metric for the Energy

Detector can be written as

$$
\mathrm{M}=\sum_{\mathrm{n}=0}^{\mathrm{N}}|\mathrm{x}(\mathrm{n})|^{2}
$$

where $\mathrm{N}$ is the size of the observation vector. The decision whether the signal is present or not is dependent on the value of $\mathrm{M}$.

\section{USRP NI 2920}

USRP NI 2920 software-programmable radio transceivers are designed for teaching and research in wireless communications. Programmable with NI LabVIEW software, the USRP hardware is easy-to-use RF platform for rapid prototyping applications such as broadcasting, physical layer communication, spectrum monitoring. With the ability to transmit and receive RF signals across a wide range of frequencies and plug-and-play MIMO support, the NI USRP enables a broad range of RF/communications applications covering common standards such as broadcast radio, digital TV, GSM Cellular, WiMax, 802.11 (Wi-Fi) and ZigBee. LabVIEW brings increased productivity with an graphical programming approach, and $\mathrm{m}$-file script compatibility enabling development of algorithms for physical layer communications [6].

Fig 3. Shows Block diagram of NI USRP 2920[6].Baseband I/Q signals are synthesized by the host computer and transmitted to the device over a standard gigabit Ethernet 


\section{International Journal of Science and Research (IJSR) \\ ISSN (Online): 2319-7064 \\ Index Copernicus Value (2013): 6.14 | Impact Factor (2015): 6.391}

connection. The digital up converter (DUC) mixes, filters, and interpolates the signal to $400 \mathrm{MS} / \mathrm{s}$. The digital-toanalog converter (DAC) converts the signal to analog. The low pass filter reduces noise and high frequency components in the signal. The mixer up converts the signals to a userspecified RF frequency. The PLL controls the VCO so that the device clocks and LO can be frequency locked to a reference signal. The transmit amplifier amplifies the signal, which is then transmitted through the antenna.

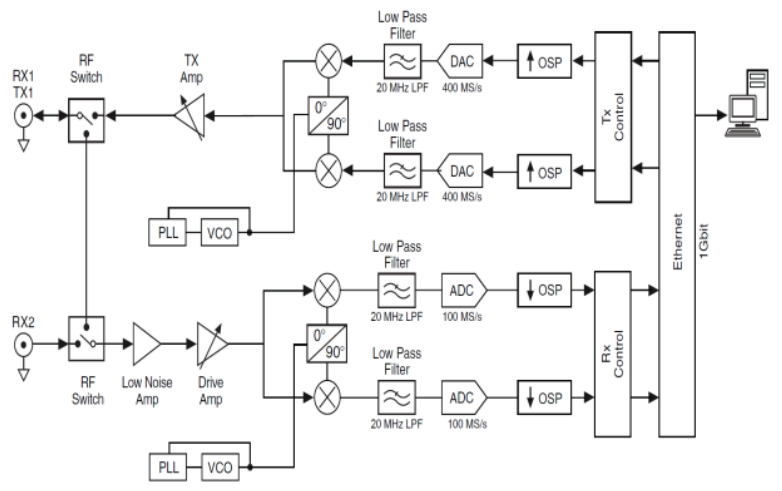

Figure 3: USRP Block Diagram [4]

The low-noise amplifier and drive amplifier amplify the incoming signal. The phase-locked loop (PLL) controls the voltage-controlled oscillator (VCO) so that the device clocks and local oscillator (LO) can be frequency locked to a reference signal. The mixer down converts the signals to the baseband in-phase (I) and quadrature-phase (Q) components. The low pass filter reduces noise and high frequency components in the signal. The analog-to-digital converter (ADC) digitizes the I and $\mathrm{Q}$ data. The digital down converter (DDC) mixes, filters, and decimates the signal to a userspecified rate. The down converted samples are passed to the host computer over a standard gigabit Ethernet connection.

Being used as a transceiver, USRP should undergo following process.

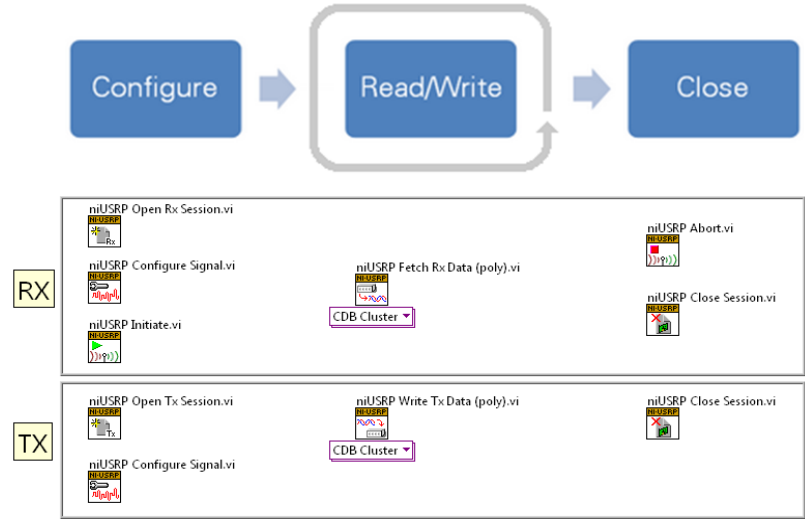

Figure 4: Eight Most-Used NI-USRP Functions [6]

Fig 4 shows the eight mostly used NI USRP functions. The mostly used blocks for transmitter are niUSRP Open Tx Session, niUSRP Configure Signal, niUSRP Write Tx Data, niUSRP Close Session. The basic blocks used in receiver are niUSRP Open Rx Session, niUSRP Configure Signal, niUSRP Initiate, niUSRP Fetch Rx Data, niUSRP Abort, niUSRP Close Session.
Table 2: Specifications of Transmitter [11]

\begin{tabular}{|l|l|}
\hline \multicolumn{1}{|c|}{ Specifications } & \multicolumn{1}{c|}{ Range } \\
\hline Frequency range & $50 \mathrm{MHz}$ to $2.2 \mathrm{GHz}$ \\
\hline Maximum Output & $50 \mathrm{MHz}$ to $1.2 \mathrm{GHz}-50 \mathrm{~mW}$ to $100 \mathrm{~mW}$ \\
\cline { 2 - 2 } Power (Pout) & $1.2 \mathrm{GHz}$ to $2.2 \mathrm{GHz}-30 \mathrm{~mW}$ to $70 \mathrm{~mW}$ \\
\hline Gain range & $0 \mathrm{~dB}$ to $31 \mathrm{~dB}$ \\
\hline
\end{tabular}

Table 2 shows the operating frequency range, Maximum output power and desired gain range when NI USRP 2920 is used as transmitter [4].

Table 3: Specifications of Receiver [11]

\begin{tabular}{|c|c|}
\hline Specifications & Range \\
\hline Frequency range & $50 \mathrm{MHz}$ to $2.2 \mathrm{GHz}$ \\
\hline Frequency step & $<1 \mathrm{kHz}$ \\
\hline Gain range & $0 \mathrm{~dB}$ to $31 \mathrm{~dB}$ \\
\hline
\end{tabular}

Table 3 shows the operating frequency range and frequency step and range of gain used when configured as receiver [6].

The LabVIEW development system provides an interface with NI USRP hardware for the development and exploration of various communications algorithms that process received signals and synthesize signals and transmit them. NI-USRP driver software provides functions (LabVIEWVIs) for the hardware/software configuration with tools for opening/closing sessions and performing $\mathrm{read} / \mathrm{write}$ operations.

On transmit side VIs, provide functionality for input data generation, channel coding, and baseband modulation. Receiver-side functionality encompasses demodulation, equalization, channel decoding, and more. With LabVIEW Modulation utilities, baseband impairments can be added, and some performance analysis such as BER measurements, modulation domain measurements, and communicationsoriented visualization. NI USRP hardware with LabVIEW software has the flexibility and functionality to deliver a platform for rapid prototyping involving signal intelligence, physical layer design, wireless signal record \& playback, algorithm validation [5].

\section{Results}

\subsection{Energy Detection}

The input data to be transmitted is generated by using the sine wave where at specific time intervals if the sample value is below threshold it is taken as digital data 0 and if above the threshold it is taken as digital data 1.The SNR value is taken for which the amplitude of the noise signal and power are calculated. The received data is obtained by adding the input data with the noise of obtained magnitude. The energy of the received signal is obtained by summing up the squared values of the samples. Threshold value of the energy is calculated by using the randomly generated values of Pf using gamma inverse function. If the detected energy value is greater than the threshold value, then it is considered as the correct value. Thus by varying the SNR value the corresponding detected count value is plotted. In Energy detection, for every $2 \mathrm{~dB}$ increase in SNR Pd increases by $10 \%$ under AWGN channel which is indicated in fig 5 . 


\section{International Journal of Science and Research (IJSR) \\ ISSN (Online): 2319-7064}

Index Copernicus Value (2013): 6.14 | Impact Factor (2015): 6.391

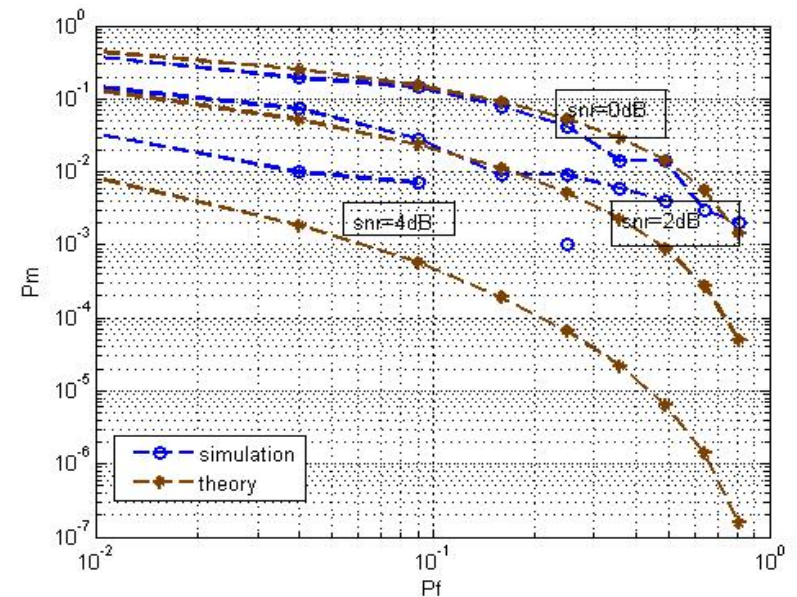

Figure 5: Complementary ROC curves for AWGN channel at different $\mathrm{SNR}$ values and $\mathrm{u}=10$.

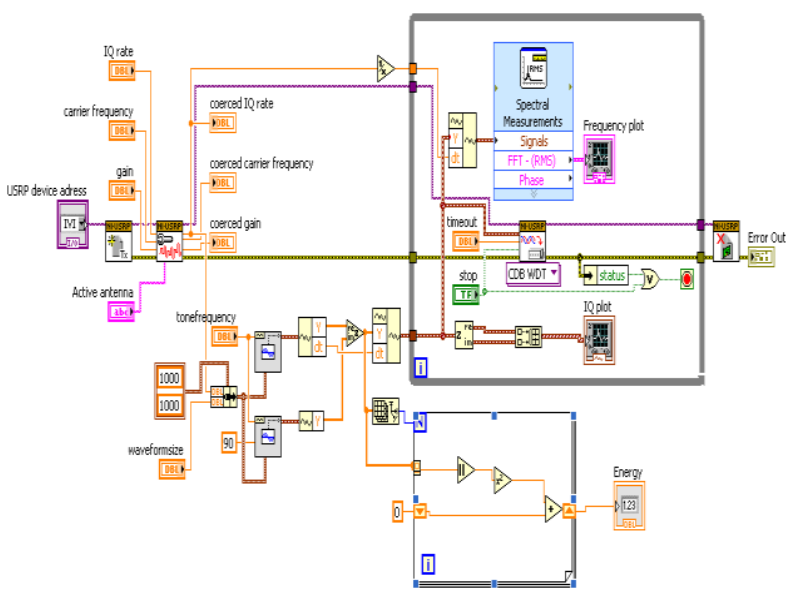

Figure 6: Block Diagram of Continuous Transmitter

Fig 6 shows the Block Diagram of Continuous Transmitter where the continuous sine waveform of $300 \mathrm{~Hz}$ frequency is converted to array of sample data which forms the real part of the data being transmitted and time difference between samples is also calculated. The same waveform is phase shifted converted and it is converted to array of sample values to form the imaginary value of the data being transmitted. The cluster consisting of elements such as starting time and time difference between samples and array of sample values is transmitted.

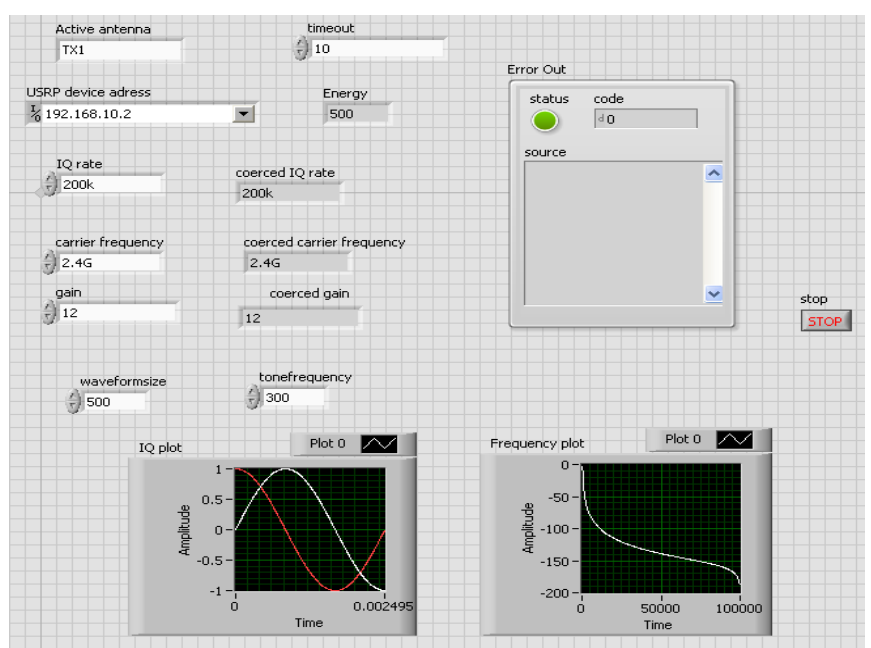

Figure 7: Front Panel of Continuous Transmitter
Fig 7.shows the Front Panel of Continuous Transmitter where the spectrum of received waveform is displayed as FFT is applied to the given cluster of data. From the array of clusters the original data is unbundled and real and imaginary part is separated and IQ plot is plotted. There is $90^{\circ}$ phase shift between the in phase and quadrature component is displayed in IQ plot.

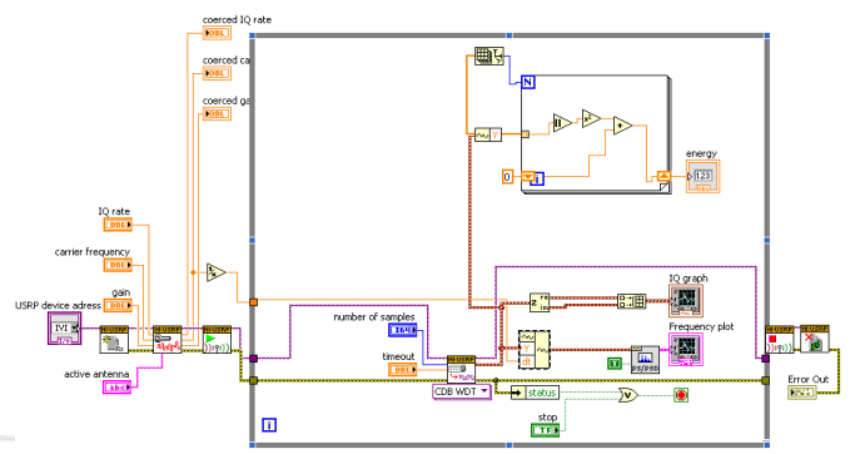

Figure 8: Block Diagram of Continuous Receiver

Fig 8 . Shows the block diagram of contiuous receiver where the the array of clusters is fetched from USRP Fetch block and FFT is taken to calculate the spectrum of the received array of data. The real and imaginary part is separated to give the IQ graph.

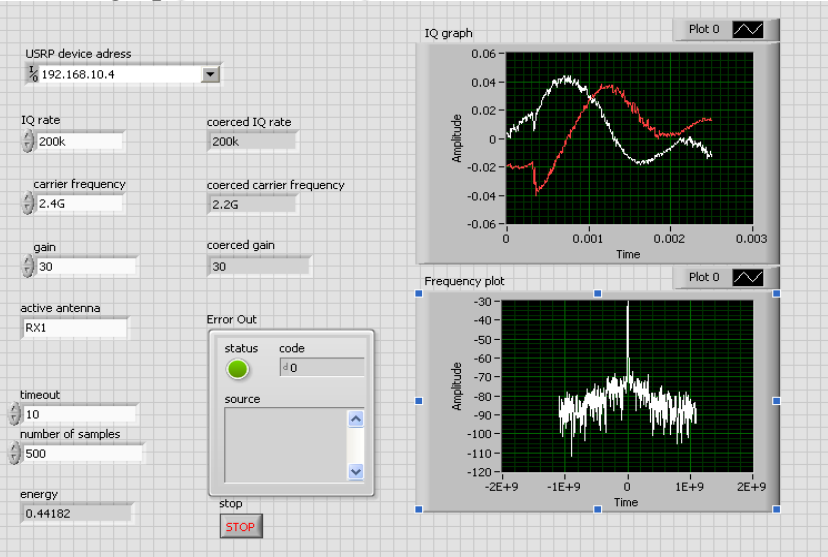

Figure 9: Front Panel of Continuous Receiver

Fig 9. Shows the Front Panel of Continuous Receiver where the device name and configuring the USRP to the desired frequency, IQ rate, antenna type and gain is configured. As the FFT for the array of data is taken the spectrum of received signal and separating into real and imaginary part is plotted as IQ plot are displayed.

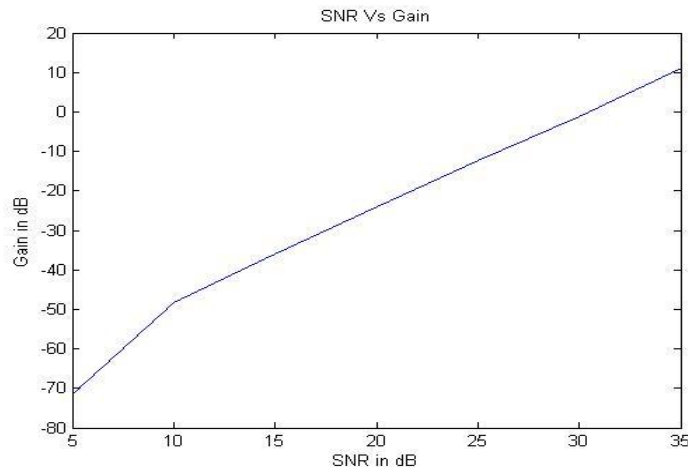

Figure 10: Gain Vs Energy 


\section{International Journal of Science and Research (IJSR) \\ ISSN (Online): 2319-7064}

Index Copernicus Value (2013): 6.14 | Impact Factor (2015): 6.391

Fig 10. Shows the plot of variation of energy with respect to gain of receiver. As the signal is transmitted through the constant gain $(12 \mathrm{~dB})$ in AWGN channel, the energy is reduced at receiver side due to noise. As the gain of receiver antenna is increased the energy of received signal also increases.

\subsection{Energy Detection With QPSK Modulation}

Fig 11. Shows the energy detection with QPSK modulation scheme with SNR $=-2 \mathrm{~dB}$. The input data to be transmitted is generated by using the sine wave where at specific time intervals and it is given to QPSK modulation block and if the sample value is below threshold it is taken as digital data 0 and if above the threshold it is taken as digital data 1.The SNR value is taken for which the amplitude of the noise signal and power are calculated. The received data is obtained by adding the input data with the noise of obtained magnitude and demodulate it. The energy of the received signal is obtained by summing up the squared values of the samples. Threshold value of the energy is calculated by using the randomly generated values of Pf using gamma inverse function. If the detected energy value is greater than the threshold value, then it is considered as the correct value.

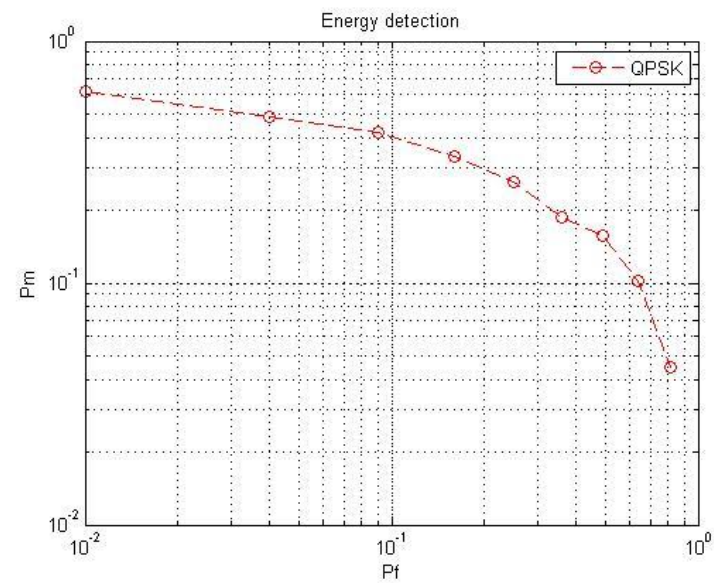

Figure 11: Complementary ROC curve for AWGN channel with QPSK modulation scheme with $\mathrm{SNR}=-2 \mathrm{~dB}$

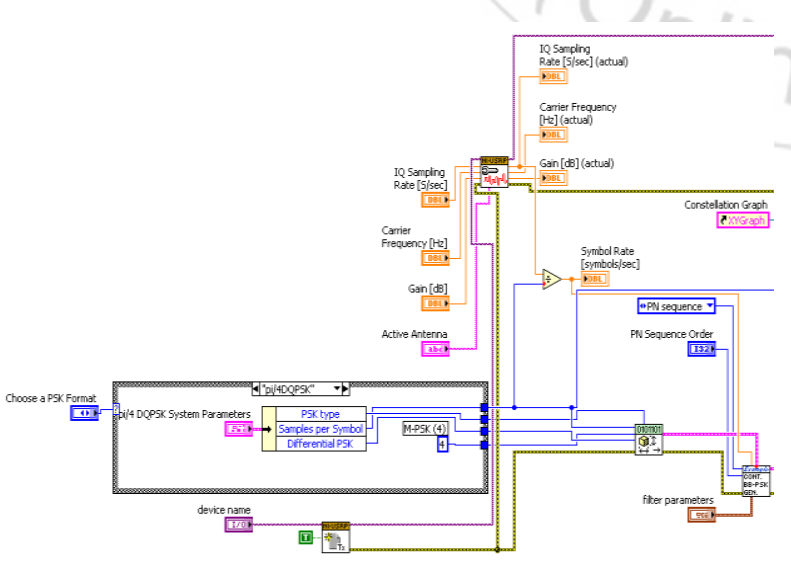

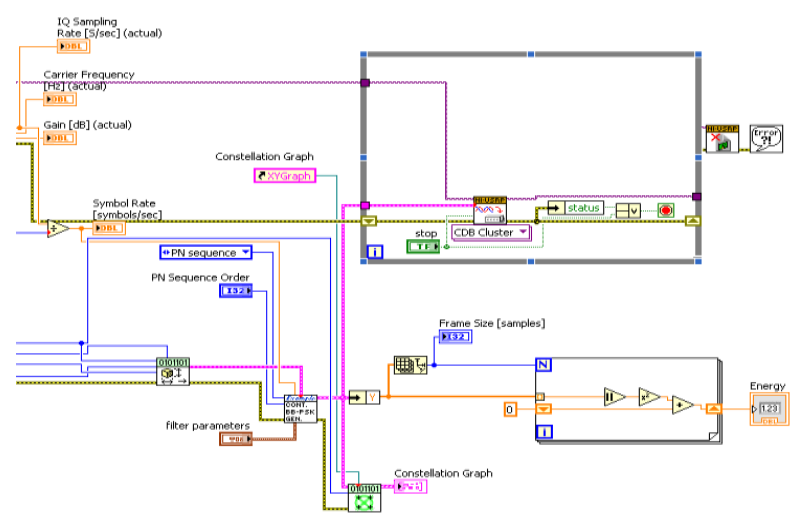

Figure 12: Block diagram of QPSK Transmitter

Fig 12. shows the block diagram of QPSK Transmitter where the samples per symbols and the PN sequence length are to be decided to give input for modulation Block as PN sequence acts as the data input .The pulse shaping filter are used to remove the abrupt change in the phase and to band limit the signal. These coefficients and QPSK modulation symbol mapped cluster are also given as input to QPSK modulation block.

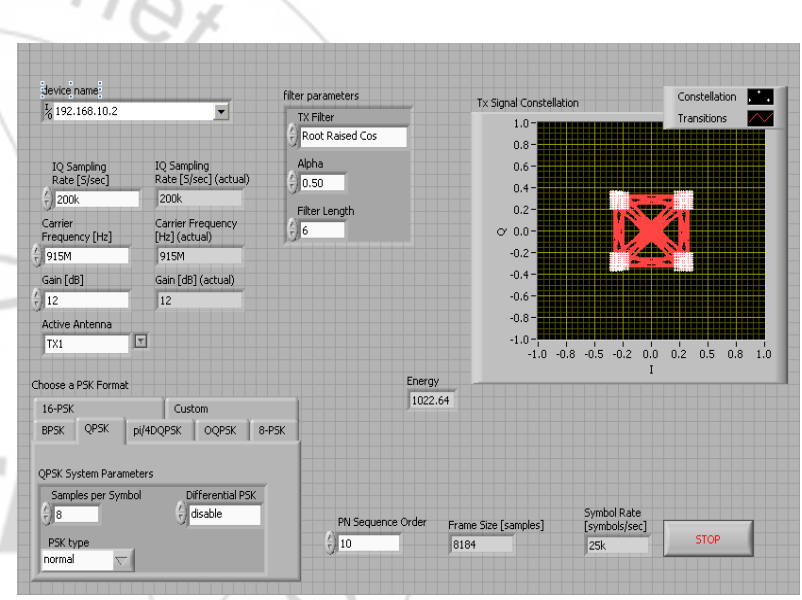

Figure 13: Front panel of QPSK Transmitter

Fig 13.shows the Front Panel of QPSK Transmitter where configuration of the transmitter is done using niUSRP configure block. From the QPSK modulation block, the output cluster is used for the Constellation plot where there is only four constellation points.The waveform of the signal and spectrum obtained by taking FFT is plotted.

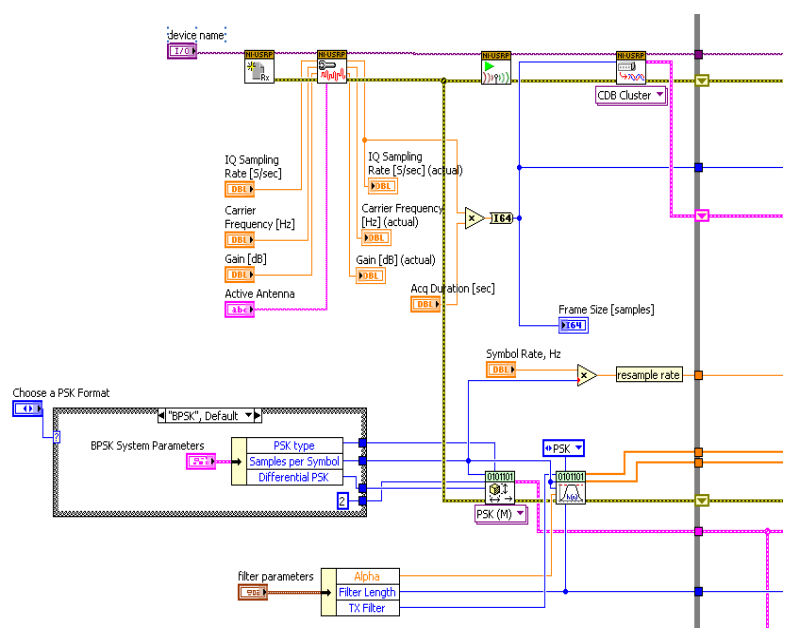




\section{International Journal of Science and Research (IJSR) \\ ISSN (Online): 2319-7064}

Index Copernicus Value (2013): 6.14 | Impact Factor (2015): 6.391

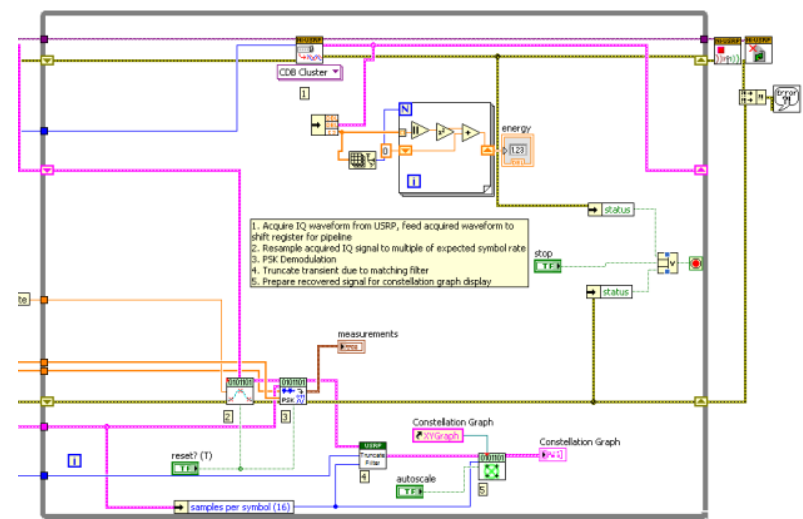

Figure 14: Block diagram of QPSK Receiver

Fig 14. shows the block diagram of QPSK Receiver where the received cluster of data is passed to the matched filter whose output is given as input to the QPSK demodulation block .the output is truncated and given as input to the constellation graph. The FFT for the received array is calculated using FFT block.

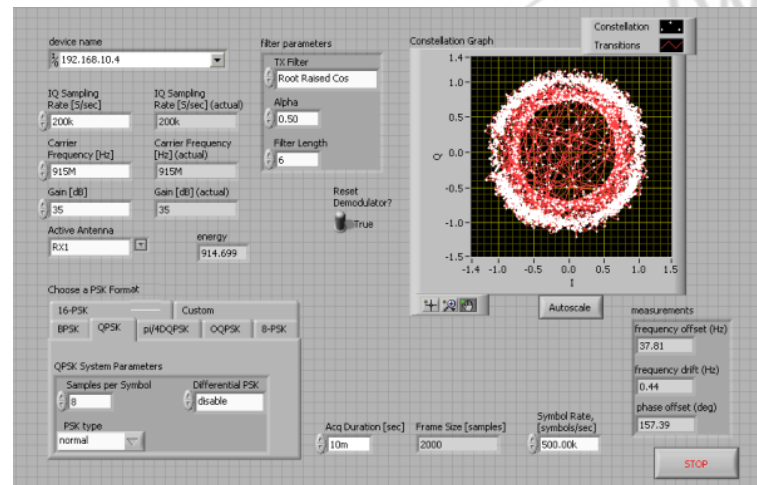

Figure 15: Front panel of QPSK Receiver

Fig 15.shows the Front Panel of QPSK Receiver where the cluster is unbundled to get the array of data and their waveform is displayed. As FFT is taken their spectrum is also plotted. The constellation graph shows that there is variation due to the channel.

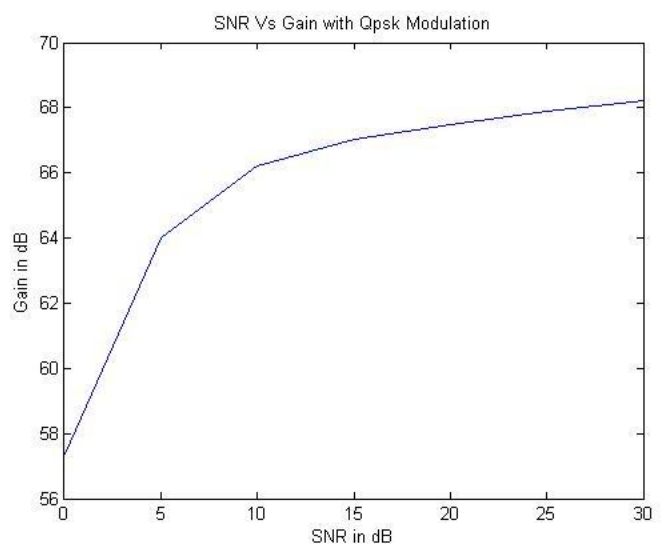

Figure 16: Gain Vs Energy with QPSK modulation

Fig 16. Shows the plot of variation of energy with respect to gain of receiver with QPSK modulation. As the signal is modulated and transmitted through the constant gain $(12 \mathrm{~dB})$ in AWGN channel, the energy is reduced at receiver side due to noise. At the receiver side, the absolute value of received signal is taken and square is taken to compute the energy and the received signal is demodulated.

\section{Conclusion}

In this paper, primary user is identified by detecting the energy of received signal using the National Instruments Universal Software Radio Peripheral Kit. The various performance measures such as constellation plot, Received waveform have been observed. We plan to implement a $2 \times 2$ Multiple Input Multiple Output system suing the National Instruments Universal Software Radio Peripheral 2920 to increase the capacity and data throughput.

\section{Acknowledgments}

We Sincerely Acknowledge UGC for the financial assistance provided under Ref. No:42-115/2013 (SR).

\section{References}

[1] Lin Gao, Xinbing Wang, YouyunXu, Qian Zhang, "Spectrum Trading in Cognitive Radio Networks: A Contract Theoretic Modeling Approach" IEEE journal on selected areas in communications, vol. 29, no. 4, April 2011.

[2] Doumi, T.L, "Spectrum considerations for public safety in the United States," IEEE Communications Magazine , vol.44, no.1, pp. 30- 37, Jan. 2006.

[3] Simon Haykin, "Cognitive Radio: Brain- Empowered Wireless Communications" IEEE journal on selected areas in communications, vol. 23, no. 2, February 2005.

[4] Federal Communications Commission, "Notice of proposed rule making and order: Facilitating opportunities for flexible, efficient, and reliable spectrum use employing cognitive radio technologies," ET Docket No. 03-108, Feb. 2005.

[5] Waleed Ejaz, "Spectrum sensing in Cognitive radio Networks", Thesis submitted to the faculty of Computer Engineering Department College of Electrical \& Mechanical Engineering, National University of Sciences and Technology, 2008.

[6] Robert W. Heath Jr, "Digital Wireless Communication Physical Layer Exploration Lab Using the NI USRP”, Manual by National Instruments.

[7] Tevfik Y"ucek and H"useyin Arslan, "A Survey of Spectrum Sensing Algorithms for Cognitive Radio Applications", IEEE Communications Surveys \& Tutorials, vol. 11, no. 1, 2009.

[8] Amir Ghasemi and Elvino S. Sousa, " Spectrum Sensing in Cognitive Radio Networks: Requirements, Challenges and Design Trade-offs", IEEE

Communications Magazine, April 2008.

[9] Mansi Subhedar And Gajanan Birajdar, " Spectrum Sensing Techniques In Cognitive Radio Networks: A Survey", International Journal Of Next-Generation Networks (Ijngn), Vol.3, No.2, June 2011.

[10] A. Rajeswari, T.G. Dhaarani, "Efficient Spectrum Sensing Methods in Cognitive Radio Networks," in Proc. IEEE Int. Conf. on Devices, Circuits and Systems. (ICDCS'12), Coimbatore, India, pp. 512-516, March 2012 and Published in IEEE Explore. 


\section{International Journal of Science and Research (IJSR) \\ ISSN (Online): 2319-7064}

Index Copernicus Value (2013): 6.14 | Impact Factor (2015): 6.391

[11] A. Rajeswari, M. Sowmiya, L. Vijayalakshmi, “Testing of digital Wireless Communication system using USRP NI 2920", International Conference on Advances in Control and Computing of Analog and Digital Systems(ACCADS 2014), Coimbatore, Dec 2014.

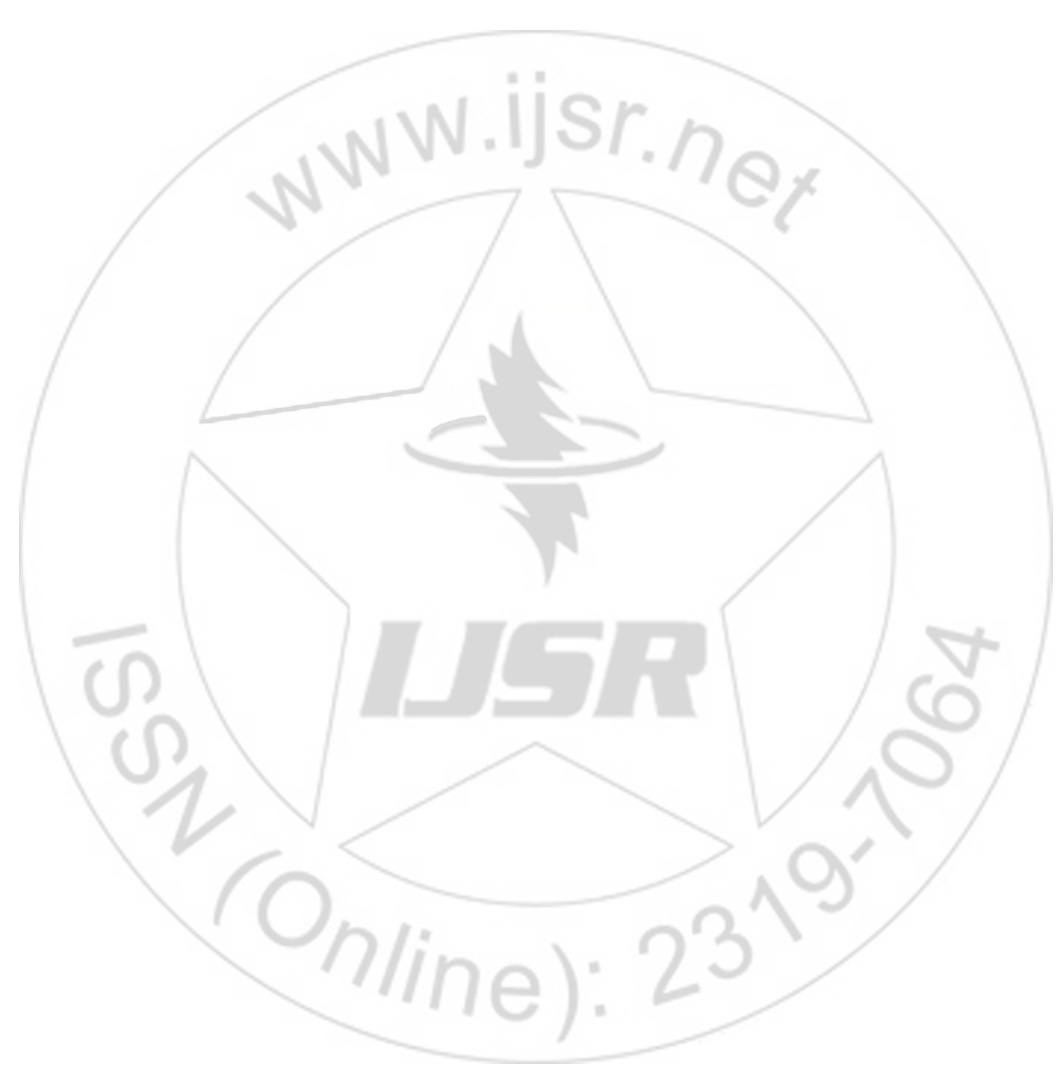

Volume 5 Issue 5, May 2016 www.ijsr.net 